\title{
Competencia léxica en inglés mediante el uso de la Estrategia Chunking Flashcards @()
}

\author{
Reading comprehension of technical texts in English and use of interactive \\ pedagogical techniques for learning in university students.
}

Zoila Victoria Herrera Andrade ${ }^{1}$, Rocío de los Ángeles Barragán Murillo. ${ }^{2}$, Esthela Isabel Colcha Guashpa ${ }^{3} \&$ Edison Hernán Salazar Calderón ${ }^{4}$

Recibido: 28-04-2019 / Revisado: 14-05-2019 /Aceptado: 30-06-2019/ Publicado: 25-07-2019

\begin{abstract}
DOI: https://doi.org/10.33262/cienciadigital.v3i3.2.715

The present research work covers about lexical competence in English using Chunking Flashcards strategy, emphasizing the importance of this study when it becomes a methodological contribution by facilitating an interactive communication between students improving oral skills to through the increase of vocabulary and in a short time. The objective was to show the incidence that it had in the development of cognitive skill. In addition, the methodology worked was explanatory method with a quail-quantitative and quantitative approach, and quasi-experimental type. The study population was 53 students at Polytechnic School of Chimborazo, divided into two groups that were duly constituted. Then, the test technique was applied with Pretest Post-test model. The questionnaire was the instrument used and applied as a test of knowledge at the beginning and at the end of intervention for both to control group and to experimental group. For validation of these instruments, the coefficient of Cronbach's Alpha was used; this allowed him to measure his level of confidence and reliability. The frequency tables determined degree of dispersion. The decisionmaking was made based on difference of existing means in the groups. It is concluded that in development of intervention during the experimentation process with application of Chunking Flashcards strategy as a new trend in the teaching English language, a high level of motivation and greater interactive participation was shown. Student Chunking, which more to increase their lexical competence improve the interest to learn the English language, and that the use of Chunking Flashcards
\end{abstract}

Escuela Superior Politécnica de Chimborazo, Docente del Centro de Idiomas ,Chimborazo Ecuador zherreraa@hotmail.com

2 Escuela Superior Politécnica de Chimborazo: Docente Inglés, Chimborazo Ecuador, obarragan@espoch.edu.ec

3 Escuela Superior Politécnica de Chimborazo: Docente de Inglés, Chimborazo Ecuador, e_colcha@espoch.edu.ec

4 Escuela Superior Politécnica de Chimborazo: Docente Inglés Chimborazo Ecuador. edisonh.salazar@espoch.edu.ec 
strategy has a positive influence on the development of lexical competence English Language.

Key Words: English Language, Lexical Competence, Cognitive Strategy, Chunking Strategy Flashcards-Pre-test Post-test.

\section{Resumen}

El presente trabajo de investigación trata sobre la competencia léxica en inglés mediante el uso de la estrategia Chunking Flashcards, se enfatiza la importancia que tiene este estudio al convertirse en un aporte metodológico por lo que permite facilitar una comunicación interactiva entre estudiantes mejorando la destreza oral a través de la incrementación de vocabulario y en corto tiempo. El objetivo es evidenciar la incidencia que tiene en el desarrollo de la destreza cognitiva. La metodología con la que se trabajó fue el método explicativo, con un enfoque cualicuanlitativo y cuantitativo, de tipo cuasi experimental. La población objeto de estudio son 53 estudiantes de la Escuela Superior Politécnica de Chimborazo, divididos en dos grupos que fueron debidamente constituidos. Se aplicó la técnica de prueba con el modelo Pre-test Post-test. El cuestionario fue el instrumento utilizado y aplicado como prueba de conocimiento al inicio y al final de la intervención tanto al grupo de control como al experimental. Para la validación de estos instrumentos se empleó el coeficiente del Alfa de Cronbach; éste permitió medir su nivel de confianza y fiabilidad. Las tablas de frecuencia determinaron el grado de dispersión. La toma de decisiones se realizó en base a la diferencia de medias existentes en los grupos. Se concluye que en el desarrollo de la intervención durante el proceso de experimentación con la aplicación de la estrategia Chunking Flaschcards como una nueva tendencia en la enseñanza del idioma ingles se evidenció un alto nivel de motivación y mayor participación interactiva docente Chunking estudiante, que a más de incrementar su competencia lexical mejoro el interés por aprender el idioma Inglés, y que el uso de la estrategia Chunking Flashcards incide positivamente en el desarrollo de la competencia léxica del Idioma Inglés.

Palabras Clave: Idioma Inglés, Competencia Léxica, Estrategía Cognitiva, Estrategía Chunking Flashcards, Pre-test - Post-test.

\section{Introducción}

El inglés se ha convertido en un idioma universal en la mayoría de países del mundo produciendo información científica y técnica a todo nivel, siendo necesario aprender esta lengua para ir a la par con el desarrollo e innovación en el contexto educativo y profesional. $\mathrm{Su}$ importancia radica en el dominio que ha alcanzado este idioma la cual viene reforzada por los cambios globales que experimenta la sociedad, ante las nuevas tendencias 
tecnológicas llegando a transformar nuestro entorno y la forma de relacionarnos con él (Importancia.org, 2015)

Menciona el Marco Común Europeo de Referencia (2002), el cómo los avances más recientes en los planteamientos científicos y curriculares sobre la enseñanza-aprendizaje de lenguas, han tendido a clarificar este estado de cosas al tomar como referencia el concepto de competencia; tal y como se puede apreciar en la legislación que regula actualmente la enseñanza de la lengua en primaria y secundaria.

Durante las últimas décadas la adquisición del léxico ha sido uno de los aspectos que ha recibido menos atención en la didáctica de las lenguas con lo que los estudios han progresado con lentitud y su enseñanza ha estado subordinada a la de las estructuras gramaticales o funcionales (Pérez Basanta, 1999).

El nivel de competencia léxica de un hablante puede ser conocido por medio del estudio de la actuación léxica, mediante la amplitud y dominio del vocabulario, y; la riqueza léxica. La primera constituye una manifestación del conocimiento que el hablante tiene de la realidad que le rodea y se relaciona con la cantidad, crecimiento y calidad del vocabulario empleado. La segunda se define a partir del número de vocablos nuevos que figuren en un texto dado, es decir; según aumente el número de vocablos en correspondencia con el crecimiento del texto, también irá creciendo el índice de riqueza léxica, aunque no al mismo ritmo (Núñez, 2010).

Maley (2004), expresa que las acciones diagnósticas servirán de base para diseñar y seleccionar actividades eficaces en las que el docente, es mediador entre el caudal léxico que posee el estudiante o aprendiz y el léxico que debe conocer al finalizar cada nivel. Tal repertorio se llega a adquirir en un proceso más asociativo y personal que lineal, puesto que depende de las experiencias del sujeto, pero también social, pues constantemente intercambiamos significados con los demás y compartimos con ellos nuestro conocimiento acerca de los significados de las palabras.

Recientemente, ha llamado la atención la aparición de los denominados chunks (trozos) de información que facilitan la comprensión y buscan el equilibrio del aprendizaje, al parecer; igual que una mirada o un tono de voz, pueden influenciar nuestras primeras impresiones sobre una persona, la percepción de una palabra puede verse afectada por ejemplo por su pronunciación, por la otra palabra que la acompaña habitualmente, por su forma en la página o en la pantalla, por asociaciones como las categorías semánticas y sintácticas a las que pertenece, por connotaciones que la palabra tiene para el aprendiz o por las circunstancias en que aparece (Fernández Smith, 2004). Ante tal circunstancia la competencia léxica desempeña una función decisiva

Para este estudio la investigación señala una metodología explicativa, con un diseño cuali cuantitativo, utilizando como instrumentos el pre y el post test aplicados en una Población 
de estudiantes dividido en dos grupos, en donde se obtuvo como resultado las diferencias y semejanzas del uso de la estrategia Chunking Flashcards y su incidencia positiva en el desarrollo de la competencia léxica del Idioma Inglés.

\section{Idioma Inglés}

El autor Muñoz (2002) define al idioma extranjero como una lengua que "no tiene presencia en la comunidad en la que vive el aprendiz. Por ejemplo, el inglés es una segunda lengua para un inmigrante mexicano en Estados Unidos, mientras que es una lengua extranjera para un estudiante en España"

La importancia de aprender un idioma se manifiesta de manera diferente en cada persona, $\mathrm{y}$; al profesorado se nos plantean muchas dudas ante el hecho educativo, lo que hace que tengamos diferentes expectativas para el grupo en la clase (Eccles y Wigfield, 1985; Good y Brophy, 2003; Jussim, Smith, Madon y Palumbo, 1998; Pellegrini y Blachtford, 2000; Rubie-Davies, 2006). Algunas veces no entendemos bien su comportamiento y la distancia que normalmente existe entre ambos, suele evitar un mejor conocimiento de sus formas de proceder y la naturaleza de éstas, lo que se convierte en un obstáculo para el buen funcionamiento de la clase de inglés. Por tanto, el idioma ingles se ha convertido en una herramienta de mucha importancia y útil en los últimos años a nivel mundial, al saber que todas las actividades y relaciones empresariales tienen como requisito tener dominio de este idioma.

\section{Elementos que implica el aprendizaje de un idioma}

Se cita lo expresado por el autor Lighbown (1993), quien manifiesta "el lenguaje es un sistema de símbolos arbitrarios, que permiten a todas las personas en una cultura determinada, u otras personas que han aprendido el sistema de esa cultura, comunicarse o interactuar". Este autor expone sobre lo importante que tiene el uso de la simbología en el aprendizaje del idioma extranjero el cual contribuye a una interacción eficiente. Es un sistema de símbolos en razón de que, si decimos, por ejemplo "El hombre" o "Los hombres", o la palabra "llegar" y "llegada ", indica significados diferentes. El orden de las palabras también es importante, por ejemplo: "El gato mordió la dama" / "La dama mordió al gato". Cada idioma opera dentro de un sistema, es decir dentro de sus propios patrones recurrentes o arreglos que sean significativas para sus hablantes.

\section{Principios del aprendizaje de un Idioma Extranjero}

La autora Sole (1993), al hablar de los principios del aprendizaje de un idioma extranjero, dice que el lenguaje hablado es fundamental, por ello es importante al momento de aprender un idioma extranjero considerar que la estructura del sistema debe ir de la mano con el sistema de sonido; los estudiantes necesitan saber las características significativas del idioma, en este caso el inglés, como el orden de palabras. Aprender un idioma significa 
adquirir la capacidad de comunicarse, formular y responder preguntas, hacer declaraciones y producir formas (palabras, frases, oraciones, etc.) normales y auténticas que son utilizados por hablantes nativos. Además, incluye aprender sobre la cultura, gestos y expresiones habladas que dan mayor significado a las palabras o frases, como fue dicho anteriormente, la connotación del mensaje.

\section{Teorías sobre el aprendizaje de una lengua}

Burrhus Skinner en su libro "Conducta Verbal" (1957), concibe el proceso de adquisición lingüística como una serie de hábitos que imitan y repiten, una y otra vez, respuestas a estímulos concretos o respuestas a asociaciones condicionadas. Él dice que el ser humano no tiene nada innato a la hora de aprender el lenguaje, ya que hay que verlo como una conducta más y, a partir de ahí, ver como se modifica.

La Teoría Innatista o de Adquisición del Lenguaje formulada por el psicolingüista Noam Chomsky (1959) a finales de los años cincuenta, afirma que los niños están biológicamente programados para el lenguaje y que las lenguas se desarrollan en el niño de la misma manera que otras funciones biológicas se desarrollan. De acuerdo a Chomsky, la adquisición del lenguaje se asemeja al proceso de aprendizaje para caminar y el medio ambiente hace una contribución básica. Su teoría surgió como una reacción a la teoría conductista del aprendizaje basado en la imitación y la formación de hábitos.

Piaget (1926), invariablemente, ha sostenido la tesis de la primacía de lo cognitivo en el hombre y de la subordinación del lenguaje al pensamiento. El pensamiento es la estructuración de las relaciones entre hombre y ambiente. Las acciones sensomotoras son la fuente del conocimiento y el organismo actúa tan pronto entra en relación con el ambiente que lo acoge. Todas las acciones iniciales son incontrolables y pasan a coordinarse en forma de esquemas, que consiste en una secuencia definida de acciones, tanto físicas como mentales, y constituye la unidad cognitiva básica. Por medio de la coordinación, los esquemas desarrollados en un tiempo definido, pasan a formar estructuras que, según el organismo se desarrolla, se convierten de estructuras instintivas en estructuras sensomotoras y en estructuras operacionales del pensamiento.

\section{Competencia Léxica}

Durante las últimas décadas la adquisición del léxico ha sido uno de los aspectos que ha recibido menos atención en la didáctica de las lenguas con lo que los estudios han progresado con lentitud y su enseñanza ha estado subordinada a la de las estructuras gramaticales o funcionales (Pérez Basanta, 1999). El autor expone que una de las razones de esta situación responde sin duda a factores lingüísticos - inherentes a la propia naturaleza del vocabulario entre los que se pueden destacar dos: el número de piezas que componen el repertorio léxico de una lengua, que es de hecho inabarcable en su totalidad, y las dificultades que conlleva su sistematización, sobre todo en comparación con los componentes fonológico y gramatical. 


\section{Aprendizaje del Léxico}

Los autores Bogaards, Zimmerman, Morante Vallejo entre otros, exponen que la enseñanza del léxico ha estado expresada hasta hace poco tiempo materializándose en los listados de palabras heredados del Método Tradicional y metodológicamente con su enseñanza subordinada a la gramática. Así mismo (Mercedes, 2010) expresa que el enfoque comunicativo identifica que es la moda actual y el sustento ideológico o conceptual en el que se basa la enseñanza de lenguas extranjeras, incluye a la conocida competencia léxica como una de las competencias esenciales.

\section{Estrategias Cognitivas}

Según Chadwick (1996), las estrategias cognitivas son procesos de dominio general para el control del funcionamiento de las actividades mentales, incluyendo las técnicas, destrezas y habilidades que la persona usa consciente o inconscientemente para manejar, controlar, mejorar y dirigir sus esfuerzos en los aspectos cognitivos, como procesamiento, atención y ejecución, en el aprendizaje.

De acuerdo a la autora Cicarelli (2018), esta manifiesta que "Estrategias cognitivas, son conductas u operaciones mentales o acciones intelectuales implementadas esencialmente para reflexionar sobre el propio aprendizaje, analizar y sintetizar lo aprendido.

\section{Pre y Post Test}

El buen desarrollo del Pre-test permite la posterior toma de decisiones en cuanto a mejorar la redacción de las preguntas, reducir la extensión de un formulario demasiado largo, mejorar los flujos de preguntas y el orden de las mismas, entre otros objetivos. (Rodriguez, M., García, F., \& García, A., 2017).

El Post-test es una encuesta que permite evaluar luego de haber realizado el cuestionario inicial, que forma parte del diseño de un cuestionario de investigación "después de", con el cual se desea responder a las interrogantes presentadas en la investigación (Hernández, 2003)

\section{Estrategia Chunking Flashcards}

La estrategia Chunking, que en español se podría traducir como técnica de troceado, fue descrita por primera vez a principios de los años 50 como conclusión de un experimento del estudioso George A. Miller. Este científico de la universidad de Harvard explicó, en esa época, una estrategia de aprendizaje fundamentada en la limitación que caracteriza a la memoria a corto plazo de un sujeto para retener un determinado número de bloques de información. El científico recogió datos cuantitativos en su estudio que describían esa limitación de la memoria de trabajo, atribuyéndole un valor de siete bloques o paquetes de información. La teoría de Miller es la que dio luz para realizar esta investigación. 
Es decir, el empleo de la estrategia Chunking es un método de presentación de información que divide los conceptos en pequeños fragmentos o trozos (chunks) de información para facilitar la comprensión al recodificar la información que tenemos agrupándola de tal manera que se pueda retener de forma más fácil y por más tiempo en la memoria.

Se conceptualiza (Chunking) que es fragmentar, esto quiere decir que es el proceso mental de dividir en partes o fragmentos (chunks) un enunciado o (AllenNewell., 1990), hace referencia diciendo "Los fragmentos son las unidades de organización de la memoria y fragmentación se refiere a la creación de dichas unidades".

El nivel de fragmentación, es decir, la cantidad de información que se almacena en un fragmento, determina la capacidad de procesamiento de las personas. (Ellis, 1996), afirma que "Mientras que la capacidad de la memoria de trabajo para guardar datos es bastante constante, su nivel de almacenamiento de información puede ser aumentado mediante la fragmentación" (p.76).

(Velasquez, 2013) Las Flashcards son fichas de aprendizaje muy efectivas a la hora de memorizar información. Cada ficha es como una tarjeta con dos caras, el frente y el revés. En el frente se escribe el vocablo, fecha, fórmula o imagen que queremos memorizar. En el revés escribiremos la traducción, pronunciación, explicación, solución al problema o detalles sobre el hecho histórico mencionado en el frente. Una vez que las fichas están listas, tan solo tenemos que coger el conjunto de fichas y partiendo de la información con la que contamos en el revés, debemos adivinar la contenida en el revés.

\section{Cómo aplicar la estrategia Chunking Flashcards}

El Chunking es uno de esos "secretos" que todos podemos utilizar para conseguir una memoria privilegiada ya sea con los estudiantes o con cualquier ser humano. A este respecto la autora (Ochaita, 1988) dice que "existe básicamente dos hipótesis generales o explicaciones alternativas. La primera de ellas considera que el crecimiento en la amplitud de memoria es debido a la adquisición de estrategias educativas, tales como repaso, agrupamiento, Chunking, estrategias de recuperación. Por lo contrario, la segunda hipótesis postula que el crecimiento en la amplitud de memoria es el resultado de una mejora en los procesos básicos o variables no estratégicas, tales como una mayor rapidez en la identificación del ítem, mayor habilidad para ordenar los ítems presentados seriamente, susceptibilidad a la interferencia, etc.".

\section{Metodología}

La metodología utilizada en esta investigación referencia al autor Tamayo (2004), quien en su libro Metodología de la Investigación, expone las directrices para llevar a la práctica este estudio. El diseño de la investigación es Cuasi experimental, para recopilar la información se basó en la técnica de pruebas, diseñando un Pre-test, como medición previa a la aplicación del tratamiento a fin de analizar el equivalente entre los grupos porque se trabaja 
con registros de control aplicados a dos grupos pequeños de estudiantes, de características comunes seleccionados de manera intencional a quienes se los denomina grupo experimental y grupo de control con el fin de verificar o comprobar las variables luego de la aplicación del Post-test.

La investigación tiene un enfoque Cuali-Cuantitativo; en donde tomamos datos para probar la hipótesis en base a la medición numérica y análisis estadístico que nos permita establecer patrones de comportamiento y comprobación de variables, así como la descripción de cualidades que se usan en el análisis de los cuestionarios (Welers, 1986). El nivel de la investigación es explicativo, su propósito es demostrar que los cambios en la variable dependiente (aprendizaje de destrezas) fueron causados por la variable independiente (recurso didáctico Chunking).

El tipo de investigación es de campo y descriptiva. Los datos se obtuvieron directamente de la fuente de los educandos del segundo nivel de inglés, y por la ayuda de docentes del Centro de Idiomas de la ESPOCH, a través de los instrumentos Pre-test y Post-test.

La experimentación duro doce horas clase por semana durante 6 semanas; se inició con los grupos experimental y de control; una vez establecidos, fueron evaluados a través de un Pretest mediante un cuestionario. Los resultados obtenidos dieron la pauta para la etapa de intervención donde se hizo la aplicación gradual y sucesiva del modelo de progresión temática derivada en las clases de inglés a fin de evaluar su efecto en la destreza de su léxico en inglés. Seguidamente, se procedió al estudio de la variable dependiente. Posterior a ello, se efectuó un análisis de cada uno de los componentes estructurales por separado para proceder a su descomposición micro estructural en oraciones y vocabulario, y; ver su léxico usando la plataforma Quizlet.

La fase de aplicación se ejecutó de manera guiada, en el periodo académico abril-agosto 2017, seleccionando su población de estudio a los estudiantes de los cursos " $\mathrm{G}$ " " $\mathrm{H}$ " y "O" del Segundo Nivel del Centro de Idiomas de la Escuela Superior Politécnica de Chimborazo, integrada por treinta y uno (31), treinta y ocho (38) y veintitrés (23) estudiantes respectivamente sumando un total de noventa y dos estudiantes.

El total de la población lo integran cincuenta y tres (53) estudiantes. Como grupo experimental se trabajó con el paralelo "O" a quienes se aplicó el CHUNKING FLASHCARD estrategia didáctica para poder determinar la influencia en el desarrollo de destrezas en el aprendizaje del inglés, se explica que el grupo de control son el paralelo " $\mathrm{H}$ " utilizando la metodología cotidiana de empleo de recurso audiovisual y la validación del instrumento lo realizó con el paralelo " $G$ " de la institucion. Al final de cada actividad se midió el nivel de logro a través de preguntas de reflexión y actividades de retroalimentación sobre el proceso para tener una mejor apreciación del nivel de logro alcanzado por los estudiantes. 
Tabla 1. Muestra Segundo Nivel del Centro de Idiomas ESPOCH

\begin{tabular}{lccc}
\hline & DOCENTES & ESTUDIANTES & PORCENTAJE \\
\hline Docente investigador & 1 & & \\
Grupo de control & & 30 & 57 \\
Grupo experimental & & 23 & 43 \\
Tota, de la muestra & 1 & 53 & 100 \\
\hline
\end{tabular}

Fuente: Secretaría del Centro de Idiomas ESPOCH

\section{Resultados y Validación}

\section{Resultados}

Tabla 2. Resumen Destrezas y Contenidos que se evalúan en el Pre-test Grupo de Control

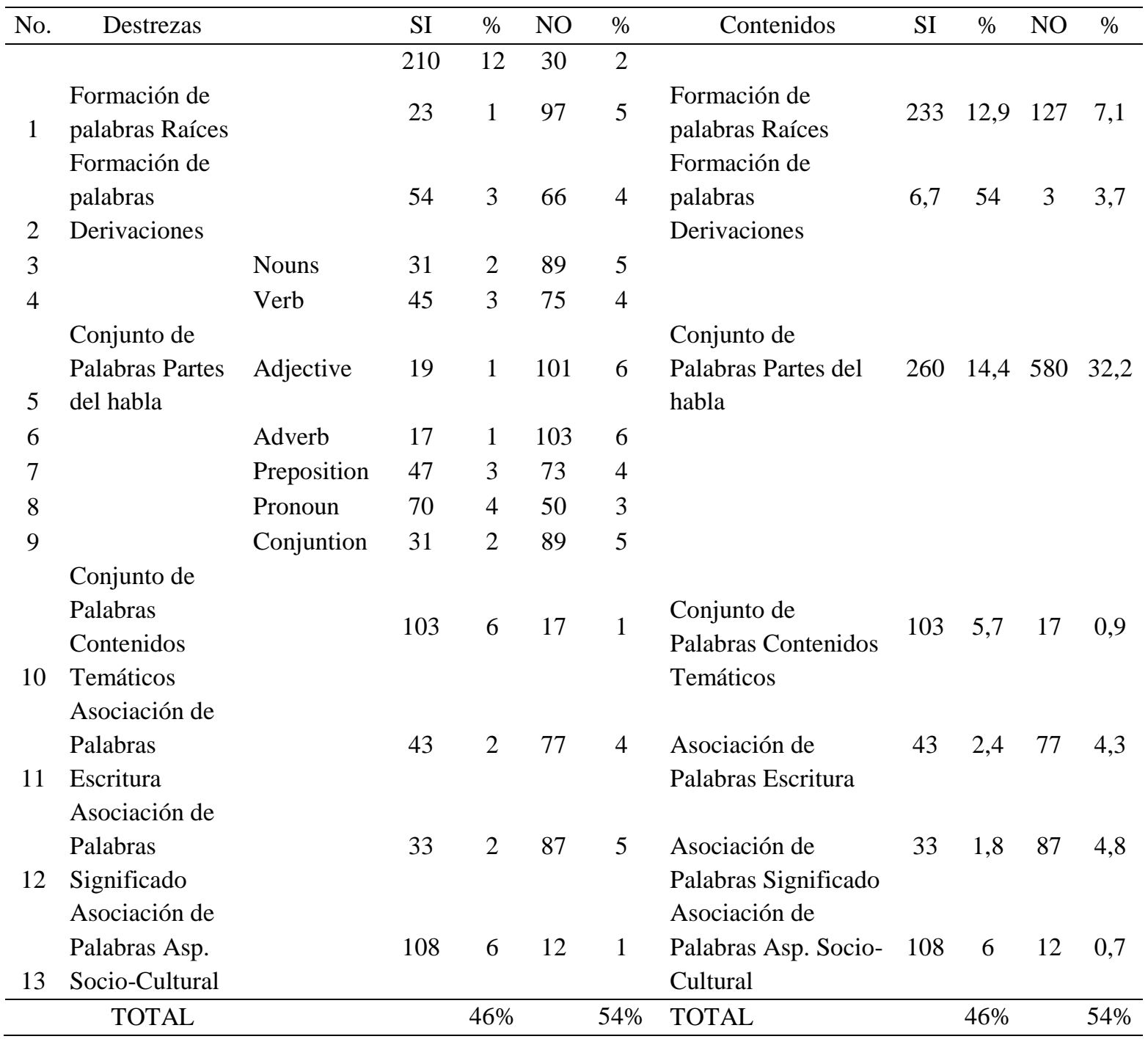


Fuente: Secretaría del Centro de Idiomas ESPOCH

Tabla 3. Resumen de Destrezas y Contenidos que se evalúan en el Pre-test Grupo Experimental

\begin{tabular}{|c|c|c|c|c|c|c|c|c|c|c|c|}
\hline No. & Destrezas & & SI & $\%$ & $\mathrm{NO}$ & $\%$ & Contenidos & SI & $\%$ & NO & $\%$ \\
\hline \multirow{4}{*}{1} & & & 171 & 12 & 13 & 1 & \multirow{4}{*}{$\begin{array}{l}\text { Formación de } \\
\text { palabras Raíces } \\
\text { Formación de } \\
\text { palabras }\end{array}$} & \multirow{4}{*}{190} & \multirow{4}{*}{13,8} & \multirow{4}{*}{86} & \multirow{4}{*}{6,2} \\
\hline & $\begin{array}{l}\text { Formación de } \\
\text { palabras }\end{array}$ & & & & & & & & & & \\
\hline & Raíces & & 19 & 1 & 73 & 5 & & & & & \\
\hline & $\begin{array}{l}\text { Formación de } \\
\text { palabras }\end{array}$ & & & & & & & & & & \\
\hline 2 & Derivaciones & & 37 & 3 & 55 & 4 & Derivaciones & 37 & 2,7 & 55 & 4 \\
\hline 3 & & Nouns & 16 & 1 & 76 & 6 & & & & & \\
\hline \multirow[t]{3}{*}{4} & & Verb & 32 & 2 & 60 & 4 & & & & & \\
\hline & Conjunto de & & & & & & \multirow{7}{*}{$\begin{array}{l}\text { Conjunto de } \\
\text { Palabras Partes } \\
\text { del habla }\end{array}$} & \multirow{7}{*}{180} & \multirow{7}{*}{13} & \multirow{7}{*}{464} & \multirow{7}{*}{33,6} \\
\hline & $\begin{array}{l}\text { Palabras } \\
\text { Partes del }\end{array}$ & Adjective & & & & & & & & & \\
\hline 5 & habla & & 20 & 1 & 72 & 5 & & & & & \\
\hline 6 & & Adverb & 14 & 1 & 78 & 6 & & & & & \\
\hline 7 & & Preposition & 32 & 2 & 60 & 4 & & & & & \\
\hline 8 & & Pronoun & 55 & 4 & 37 & 3 & & & & & \\
\hline \multirow[t]{3}{*}{9} & & Conjuntion & 11 & 1 & 81 & 6 & & & & & \\
\hline & Conjunto de & & & & & & Conjunto de & \multirow[b]{2}{*}{77} & \multirow[b]{2}{*}{5,6} & \multirow{3}{*}{15} & \multirow{3}{*}{1,1} \\
\hline & $\begin{array}{l}\text { Palabras } \\
\text { Contenidos }\end{array}$ & & & & & & $\begin{array}{l}\text { Palabras } \\
\text { Contenidos }\end{array}$ & & & & \\
\hline \multirow[t]{3}{*}{10} & Temáticos & & 77 & 6 & 15 & 1 & Temáticos & & & & \\
\hline & Asociación de & & & & & & Asociación de & & & & \\
\hline & Palabras & & & & & & Palabras & 29 & 2,1 & 63 & 4,6 \\
\hline \multirow[t]{2}{*}{11} & Escritura & & 29 & 2 & 63 & 5 & Escritura & & & & \\
\hline & $\begin{array}{l}\text { Asociación de } \\
\text { Palabras }\end{array}$ & & & & & & $\begin{array}{l}\text { Asociación de } \\
\text { Palabras }\end{array}$ & 30 & 2,2 & 62 & 4,5 \\
\hline \multirow[t]{3}{*}{12} & Significado & & 30 & 2 & 62 & 4 & Significado & & & & \\
\hline & Asociación de & & & & & & Asociación de & & & & \\
\hline & Palabras Asp. & & & & & & Palabras Asp. & 92 & 6,7 & 0 & 0 \\
\hline \multirow[t]{2}{*}{13} & Socio-Cultural & & 92 & 46 & 0 & 0 & Socio-Cultural & & & & \\
\hline & TOTAL & & 635 & $46 \%$ & 745 & $54 \%$ & TOTAL & 635 & $46 \%$ & 745 & $54 \%$ \\
\hline
\end{tabular}

Fuente: Secretaría del Centro de Idiomas ESPOCH

Tabla 4. Resumen Destrezas y Contenidos que se evalúan en el Post-test Grupo de Control

\begin{tabular}{|c|c|c|c|c|c|c|c|c|c|c|}
\hline No. & Destrezas & SI & $\%$ & NO & $\%$ & Contenidos & SI & $\%$ & $\mathrm{NO}$ & $\%$ \\
\hline & & 187 & 10 & 53 & 3 & & & & & \\
\hline & $\begin{array}{l}\text { Formación de } \\
\text { palabras }\end{array}$ & 78 & 4 & 42 & 2 & $\begin{array}{l}\text { Formación de } \\
\text { palabras }\end{array}$ & 265 & 14,7 & 95 & 5,3 \\
\hline 1 & Raíces & & & & & Raíces & & & & \\
\hline 2 & $\begin{array}{l}\text { Formación de } \\
\text { palabras } \\
\text { Derivaciones }\end{array}$ & 58 & 3 & 62 & 3 & $\begin{array}{l}\text { Formación de } \\
\text { palabras } \\
\text { Derivaciones }\end{array}$ & 58 & 3,2 & 62 & 3,4 \\
\hline
\end{tabular}


3

4

Conjunto de

Palabras

Partes del

habla

Conjunto de

Palabras

Contenidos

10 Temáticos

Asociación de

Palabras

11 Escritura

Asociación de

Palabras

12 Significado

Asociación de

Palabras Asp.

Socio-

13 Cultural

$\begin{array}{lllll}\text { Nouns } & 75 & 4 & 45 & 3 \\ \text { Verb } & 68 & 4 & 52 & 3\end{array}$

Conjunto de

$\begin{array}{llllllllll}\text { Adjective } & 69 & 4 & 51 & 3 & \text { Palabras Partes } & 446 & 24,8 & 394 & 21,9\end{array}$

del habla

$\begin{array}{lllll}\text { Adverb } & 56 & 3 & 64 & 4 \\ \text { Preposition } & 80 & 4 & 40 & 2 \\ \text { Pronoun } & 42 & 2 & 78 & 4 \\ \text { Conjuntion } & 56 & 3 & 64 & 4\end{array}$

Conjunto de

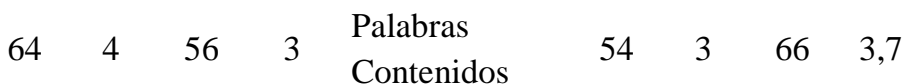

Temáticos

Asociación de

$\begin{array}{lllllllll}46 & 3 & 74 & 4 & \text { Palabras } & 46 & 2,6 & 74 & 4,1\end{array}$

Escritura

Asociación de

$\begin{array}{lllllllll}74 & 4 & 46 & 3 & \text { Palabras } & 74 & 4,1 & 46 & 2,6\end{array}$

Significado

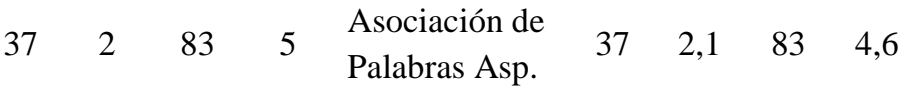

Socio-Cultural

\begin{tabular}{llllllll} 
TOTAL & $55 \%$ & $45 \%$ & TOTAL & 980 & $54 \%$ & 820 & $46 \%$ \\
\hline
\end{tabular}

Fuente: Secretaría del Centro de Idiomas ESPOCH

Tabla 5. Resumen Destrezas y Contenidos que se evalúan en el Post-test Grupo Experimental

\begin{tabular}{|c|c|c|c|c|c|c|c|c|c|c|c|}
\hline No. & Destrezas & & SI & $\%$ & NO & $\%$ & Contenidos & SI & $\%$ & $\mathrm{NO}$ & $\%$ \\
\hline & & & 160 & 12 & 24 & 2 & & & & & \\
\hline 1 & $\begin{array}{l}\text { Formación de } \\
\text { palabras Raíces }\end{array}$ & & 86 & 6 & 6 & 0 & $\begin{array}{l}\text { Formación de } \\
\text { palabras Raíces }\end{array}$ & 246 & 17,8 & 30 & 2,2 \\
\hline & $\begin{array}{l}\text { Formación de } \\
\text { palabras }\end{array}$ & & 84 & 6 & 8 & 1 & $\begin{array}{l}\text { Formación de } \\
\text { palabras }\end{array}$ & 84 & 6,1 & 8 & 0,6 \\
\hline 2 & Derivaciones & & & & & & Derivaciones & & & & \\
\hline 3 & & Nouns & 85 & 6 & 7 & 1 & & & & & \\
\hline 4 & & Verb & 83 & 6 & 9 & 1 & & & & & \\
\hline & Conjunto de & & & & & & Conjunto de & & & & \\
\hline 5 & $\begin{array}{l}\text { Palabras Partes } \\
\text { del habla }\end{array}$ & Adjective & 77 & 6 & 15 & 1 & $\begin{array}{l}\text { Palabras Partes } \\
\text { del habla }\end{array}$ & 540 & 39,1 & 104 & 7,5 \\
\hline 6 & & Adverb & 73 & 5 & 19 & 1 & & & & & \\
\hline 7 & & Preposition & 77 & 6 & 15 & 1 & & & & & \\
\hline 8 & & Pronoun & 74 & 5 & 18 & 1 & & & & & \\
\hline 9 & & Conjuntion & 71 & 5 & 21 & 2 & & & & & \\
\hline & Conjunto de & & & & & & Conjunto de & & & & \\
\hline 10 & $\begin{array}{l}\text { Palabras } \\
\text { Contenidos } \\
\text { Temáticos }\end{array}$ & & 78 & 6 & 14 & 1 & $\begin{array}{l}\text { Palabras } \\
\text { Contenidos } \\
\text { Temáticos }\end{array}$ & 78 & 5,7 & 14 & 1 \\
\hline
\end{tabular}




\begin{tabular}{|c|c|c|c|c|c|c|c|c|c|c|}
\hline & $\begin{array}{l}\text { Asociación de } \\
\text { Palabras }\end{array}$ & 85 & 6 & 7 & 1 & $\begin{array}{l}\text { Asociación de } \\
\text { Palabras }\end{array}$ & 85 & 6,2 & 7 & 0,5 \\
\hline 11 & Escritura & & & & & Escritura & & & & \\
\hline & $\begin{array}{l}\text { Asociación de } \\
\text { Palabras }\end{array}$ & 89 & 6 & 3 & 0 & $\begin{array}{l}\text { Asociación de } \\
\text { Palabras }\end{array}$ & 89 & 6,4 & 3 & 0,2 \\
\hline 12 & Significado & & & & & Significado & & & & \\
\hline 13 & $\begin{array}{l}\text { Asociación de } \\
\text { Palabras Asp. } \\
\text { Socio-Cultural }\end{array}$ & 78 & 6 & 14 & 1 & $\begin{array}{l}\text { Asociación de } \\
\text { Palabras Asp. } \\
\text { Socio-Cultural }\end{array}$ & 78 & 5,7 & 14 & 1 \\
\hline & TOTAL & 1200 & $87 \%$ & 180 & $13 \%$ & TOTAL & 1200 & $87 \%$ & 180 & $13 \%$ \\
\hline
\end{tabular}

Fuente: Secretaría del Centro de Idiomas ESPOCH

ESPOCH

\section{Validación}

Expuesto en forma de resumen la aplicación del Pre-test y Post-test, se procede al análisis de la información obtenida mediante el diseño cuasiexperimental en donde se pudo realizar diversos análisis estadísticos como el análisis de varianza, la prueba "t" entre otras, así la prueba de la hipótesis general de investigación se realizó con "t-student" para valorar si hay diferencias significativas puesto que se trató de una hipótesis de diferencia entre grupos, cuya fórmula fue la siguiente.

$$
\mathrm{t}=\frac{\overline{\mathrm{X}}-\overline{\mathrm{Y}}}{\sqrt{\frac{(\mathrm{n}-1) \mathrm{S}_{1}^{2}+(\mathrm{m}-1) \mathrm{S}_{2}^{2}}{\mathrm{n}+\mathrm{m}-2} \sqrt{\frac{1}{\mathrm{n}}+\frac{1}{\mathrm{~m}}}}}
$$

Fuente: (Abraira, A. V., Pérez de Vargas, 1996)

Para poder concretar el análisis se partió de las calificaciones obtenidas en cada grupo, con esta información se elaboraron tablas estadísticas la primera para tabular en porcentajes los ítems respondidos según el grado de satisfacción de la respuesta, una segunda tabla que agrupa en porcentajes los puntajes obtenidos por destrezas y una tercera tabla resumen de calificaciones por destrezas evaluadas.

Tabla 6. Tabla de frecuencias para medidas de tendencia central pre test grupo de control

\begin{tabular}{cccccc}
\hline No. & $\begin{array}{c}\text { Clases o } \\
\text { Grupos } \\
\mathrm{xi}\end{array}$ & $\begin{array}{c}\text { Frecuencia } \\
\text { Absoluta } \\
\mathrm{Fi}\end{array}$ & $\begin{array}{c}\text { Frecuencia } \\
\text { Absoluta } \\
\text { Acumulada }\end{array}$ & $\begin{array}{c}\text { Frecuencia } \\
\text { Relativa }\end{array}$ & $\begin{array}{c}\text { Frecuencia } \\
\text { relativa } \\
\text { acumulada }\end{array}$ \\
\hline 1 & 3 & 5 & 5 & 0,17 & 0,17 \\
2 & 4 & 10 & 15 & 0,33 & 0,50 \\
3 & 5 & 10 & 25 & 0,33 & 0,83 \\
4 & 7 & 4 & 29 & 0,13 & 0,97 \\
5 & 8 & 1 & 30 & 0,03 & 1,00 \\
\hline
\end{tabular}

Fuente: Secretaría del Centro de Idiomas ESPOCH 
Media $\bar{x}=\frac{1}{n} \sum_{\mathrm{i}=1}^{\mathrm{n}} \mathrm{xi}=\frac{\mathrm{x}_{1}+\mathrm{x}_{2}+\ldots+\mathrm{x}_{\mathrm{n}}}{\mathrm{n}}=\frac{\sum \mathrm{xi}}{\mathrm{n}}=4.7$

Moda Mo $=$ Valor que más se repite $=4$

Mediana Me $=(\mathrm{n}+1) / 2=5$

Rango $\mathrm{R}=\mathrm{X}_{\text {máximo }}-\mathrm{X}_{\text {mínimo }}=5$

Tabla 7. Tabla de frecuencia para medidas de dispersión pre test grupo de control

\begin{tabular}{ccccccc}
\hline No. & $\mathrm{xi}$ & $\mathrm{fi}$ & $\mathrm{xi}{ }^{*} \mathrm{fi}$ & $\mathrm{d}$ & $\mathrm{d}^{\wedge} 2$ & $\mathrm{fi}^{*} \mathrm{~d}^{\wedge} 2$ \\
\hline 1 & 3 & 5 & 15 & $-1,7$ & 2,89 & 14,45 \\
2 & 4 & 10 & 40 & $-0,7$ & 0,49 & 4,90 \\
3 & 5 & 10 & 50 & 0,3 & 0,09 & 0,90 \\
4 & 7 & 4 & 28 & 2,3 & 5,29 & 21,16 \\
5 & 8 & 1 & 8 & 3,3 & 10,89 & 10,89 \\
\hline TOTAL & 30 & 141 & & & 52,30 \\
\hline
\end{tabular}

Fuente: Secretaría del Centro de Idiomas ESPOCH

$\operatorname{Varianza} \sigma_{\mathrm{n}}^{2}=\sum_{\mathrm{i}=1}^{\mathrm{n}}(\mathrm{xi}-\overline{\mathrm{x}})^{2}=\frac{\sum \mathrm{fi} * \mathrm{~d}^{2}}{\mathrm{~N}}=1.74333 \ldots$

Desviación Estándar $\sigma=\sqrt{\frac{\sum \mathrm{fi} * \mathrm{~d}^{2}}{\mathrm{n}}}=1.3203$

Coeficiente de variación $\mathrm{C}_{\mathrm{v}}=\frac{\sigma}{\overline{\mathrm{x}}}=28 \%$

$\overline{\mathrm{X}}+\sigma ; \overline{\mathrm{X}}-\sigma$

$6.0 ; 3.4$

Como se puede ver el mayor número de calificaciones cae dentro del rango que va desde 6.0 puntos hacia arriba hasta 3.4 puntos hacia abajo; puesto que la desviación estándar 1.32 representa el grado de dispersión que existe entre los valores de la variable con la media aritmética.

Tabla 8. Tabla de frecuencias para medidas de tendencia central pre test grupo experimental

\begin{tabular}{cccccc}
\hline No. & $\begin{array}{c}\text { Clases o } \\
\text { Grupos } \\
\mathrm{xi}\end{array}$ & $\begin{array}{c}\text { Frecuencia } \\
\text { Absoluta } \\
\mathrm{Fi}\end{array}$ & $\begin{array}{c}\text { Frecuencia } \\
\text { absoluta } \\
\text { acumulada }\end{array}$ & $\begin{array}{c}\text { Frecuencia } \\
\text { relativa }\end{array}$ & $\begin{array}{c}\text { Frecuencia } \\
\text { Relativa } \\
\text { Acumulada }\end{array}$ \\
\hline 1 & 3 & 4 & 4 & 0,17 & 0,17 \\
2 & 4 & 10 & 14 & 0,43 & 0,61 \\
3 & 5 & 4 & 18 & 0,17 & 0,78 \\
4 & 6 & 1 & 19 & 0,04 & 0,83 \\
5 & 7 & 4 & 23 & 0,17 & 1,00 \\
\hline TOTAL & & 23 & 78 & 1,00 & \\
\hline
\end{tabular}

Fuente: Secretaría del Centro de Idiomas ESPOCH

Media $\bar{x}=\frac{1}{n} \sum_{i=1}^{n} x i=\frac{x_{1}+x_{2}+\ldots+x_{n}}{n}=\frac{\sum x i}{n}=4.6086$ 
Moda Mo $=$ Valor que más se repite $=4$

Mediana $\mathrm{Me}=(\mathrm{n}+1) / 2=4$

Rango $\mathrm{R}=\mathrm{X}_{\text {máximo }}-\mathrm{X}_{\text {mínimo }}=4$

Tabla 9. Tabla de frecuencias para medidas de dispersión pre test grupo experimental

\begin{tabular}{ccccccc}
\hline No. & $\mathrm{xi}$ & $\mathrm{fi}$ & $\mathrm{xi} * \mathrm{fi}$ & $\mathrm{d}$ & $\mathrm{d}^{\wedge} 2$ & $\mathrm{fi}^{*} \mathrm{~d}^{\wedge} 2$ \\
\hline 1 & 3 & 4 & 12 & $-1,6$ & 2,59 & 10,35 \\
2 & 4 & 10 & 40 & 4,0 & 16,00 & 160,00 \\
3 & 5 & 4 & 20 & 1,0 & 1,00 & 4,00 \\
4 & 6 & 1 & 6 & 2,0 & 4,00 & 4,00 \\
5 & 7 & 4 & 28 & 3,0 & 9,00 & 36,00 \\
\hline TOTAL & & 23 & 25 & & & 214,35 \\
\hline
\end{tabular}

Fuente: Secretaría del Centro de Idiomas ESPOCH

Varianza $\sigma_{\mathrm{n}}^{2}=\sum_{\mathrm{i}=1}^{\mathrm{n}}(\mathrm{xi}-\overline{\mathrm{x}})^{2}=\frac{\sum \mathrm{fi} * \mathrm{~d}^{2}}{\mathrm{~N}}=1.74333 \ldots$

Desviación Estándar $\sigma=\sqrt{\frac{\sum \mathrm{fi} * \mathrm{~d}^{2}}{\mathrm{n}}}=1.3203$

Coeficiente de variación $\mathrm{C}_{\mathrm{v}}=\frac{\sigma}{\overline{\mathrm{X}}}=28 \%$

$\overline{\mathrm{X}}+\sigma ; \overline{\mathrm{X}}-\sigma$

$7.7 ; 1.6$

\section{Presentación de resultados del post test aplicado al grupo de control}

Como se puede ver el mayor número de calificaciones cae dentro del rango que va desde 7.7 puntos hacia arriba hasta 1.6 puntos hacia abajo; puesto que la desviación estándar 3.05 representa el grado de dispersión que existe entre los valores de la variable con la media aritmética.

Tabla 10. Tabla de frecuencias para medidas de tendencia central post test grupo de control

\begin{tabular}{cccccc}
\hline No. & $\begin{array}{c}\text { Clases o } \\
\text { Grupos } \\
\text { xi }\end{array}$ & $\begin{array}{c}\text { Frecuencia } \\
\text { Absoluta } \\
\mathrm{Fi}\end{array}$ & $\begin{array}{c}\text { Frecuencia } \\
\text { absoluta } \\
\text { acumulada }\end{array}$ & $\begin{array}{c}\text { Frecuencia } \\
\text { relativa }\end{array}$ & $\begin{array}{c}\text { Frecuencia } \\
\text { Relativa } \\
\text { Acumulada }\end{array}$ \\
\hline 1 & 4 & 7 & 7 & 0,23 & 0,23 \\
2 & 5 & 9 & 16 & 0,30 & 0,53 \\
3 & 6 & 8 & 24 & 0,27 & 0,80 \\
4 & 7 & 5 & 29 & 0,17 & 0,97 \\
5 & 8 & 1 & 30 & 0,03 & 1,00 \\
\hline TOTAL & 30 & & 1,00 & \\
\hline
\end{tabular}

Fuente: Secretaría del Centro de Idiomas ESPOCH

Media $\bar{x}=\frac{1}{n} \sum_{i=1}^{n} x i=\frac{x_{1}+x_{2}+\cdots+x_{n}}{n}=\frac{\sum x_{i}}{n}=5.5$

Moda $=$ Valor que más se repite $=5$

Mediana $\mathrm{Me}=(\mathrm{n}+1) / 2=5$

Rango $\mathrm{R}=\mathrm{x}_{\text {máximo }}-\mathrm{x}_{\text {mínimo }}=4$

Tabla 11. Tabla de frecuencias para medidas de dispersión post test grupo de control

\begin{tabular}{ccccccc}
\hline No. & $\mathrm{xi}$ & $\mathrm{Fi}$ & $\mathrm{xi} * \mathrm{fi}$ & $\mathrm{d}$ & $\mathrm{d}^{\wedge} 2$ & $\mathrm{fi}^{*} \mathrm{~d}^{\wedge} 2$ \\
\hline 1 & 4 & 7 & 28 & $-1,5$ & 2,15 & 15,06 \\
2 & 5 & 9 & 45 & $-0,5$ & 0,22 & 1,96 \\
3 & 6 & 8 & 48 & 0,5 & 0,28 & 2,28 \\
4 & 7 & 5 & 35 & 1,5 & 2,35 & 11,76 \\
5 & 8 & 1 & 8 & 2,5 & 6,42 & 6,42
\end{tabular}


Fuente: Secretaría del Centro de Idiomas ESPOCH

Varianza $\sigma_{\mathrm{n}}^{2}=\sum_{\mathrm{i}=1}^{\mathrm{n}}(\mathrm{xi}-\overline{\mathrm{x}})^{2}=\frac{\sum \mathrm{fi} \times \mathrm{d}^{2}}{\mathrm{~N}}=1,2488889$

Desviación Estándar $\sigma=\sqrt{\frac{\sum \mathrm{fi} \times \mathrm{d}^{2}}{\mathrm{n}}}=1,117537$

Coeficiente de Variación $C_{v}=\frac{\sigma}{\bar{x}}=20 \%$

$\overline{\mathrm{x}}+\sigma ; \overline{\mathrm{x}}-\sigma$

6,$6 ; 4,3$
Como se puede notar el mayor número de calificaciones cae dentro del rango que va desde 6.6 puntos hacia arriba hasta 4.3 puntos hacia abajo; puesto que la desviación estándar 1.12 representa el grado de dispersión que existe entre los valores de la variable con la media aritmética.

\section{Presentación de resultados del Post-test aplicado al grupo experimental}

Tabla 12. Tabla de frecuencias para medidas de tendencia central post-test grupo experimental

\begin{tabular}{cccccc}
\hline No. & $\begin{array}{c}\text { Clases o } \\
\text { Grupos } \\
\mathrm{xi}\end{array}$ & $\begin{array}{c}\text { Frecuencia } \\
\text { Absoluta } \\
\mathrm{Fi}\end{array}$ & $\begin{array}{c}\text { Frecuencia } \\
\text { absoluta } \\
\text { acumulada }\end{array}$ & $\begin{array}{c}\text { Frecuencia } \\
\text { Relativa }\end{array}$ & $\begin{array}{c}\text { Frecuencia } \\
\text { Relativa } \\
\text { Acumulada }\end{array}$ \\
\hline 1 & 6 & 1 & 1 & 0,04 & 0,04 \\
2 & 7 & 1 & 2 & 0,04 & 0,09 \\
3 & 8 & 5 & 7 & 0,22 & 0,30 \\
4 & 9 & 12 & 19 & 0,52 & 0,83 \\
5 & 10 & 4 & 23 & 0,17 & 1,00 \\
\hline
\end{tabular}

Fuente: Secretaría del Centro de Idiomas ESPOCH

Media $\bar{x}=\frac{1}{n} \sum_{i=1}^{n} x i=\frac{x_{1}+x_{2}+\cdots+x_{n}}{n}=\frac{\sum x_{i}}{n}=8.7$

Moda $=$ Valor que más se repite $=9$

Mediana $\mathrm{Me}=(\mathrm{n}+1) / 2=9$

Rango $\mathrm{R}=\mathrm{x}_{\text {máximo }}-\mathrm{x}_{\text {mínimo }}=4$

Tabla 131. Tabla de frecuencias para medidas de dispersión post test grupo experimental

\begin{tabular}{ccccccc}
\hline No. & $\mathrm{Xi}$ & $\mathrm{fi}$ & $\mathrm{xi} * \mathrm{fi}$ & $\mathrm{d}$ & $\mathrm{d}^{\wedge} 2$ & $\mathrm{fi} * \mathrm{~d}^{\wedge} 2$ \\
\hline 1 & 6 & 1 & 6 & $-2,7$ & 7,50 & 7,50 \\
2 & 7 & 1 & 7 & $-1,7$ & 3,02 & 3,02 \\
3 & 8 & 5 & 40 & $-0,7$ & 0,55 & 2,73 \\
4 & 9 & 12 & 108 & 0,3 & 0,07 & 0,82 \\
5 & 10 & 4 & 40 & 1,3 & 1,59 & 6,36 \\
\hline & TOTAL & 23 & 201 & & & 20,43 \\
\hline
\end{tabular}

Fuente: Secretaría del Centro de Idiomas ESPOCH 
Varianza $\sigma_{\mathrm{n}}^{2}=\sum_{\mathrm{i}=1}^{\mathrm{n}}(\mathrm{xi}-\overline{\mathrm{x}})^{2}=\frac{\sum \mathrm{fi} \times \mathrm{d}^{2}}{\mathrm{~N}}=0,8884688$

Desviación Estándar $\sigma=\sqrt{\frac{\sum \mathrm{fi} \times \mathrm{d}^{2}}{\mathrm{n}}}=0,9425862$

Coeficiente de Variación $\mathrm{C}_{\mathrm{v}}=\frac{\sigma}{\overline{\mathrm{x}}}=11 \%$

$\overline{\mathrm{x}}+\sigma ; \overline{\mathrm{x}}-\sigma$

9,$7 ; 7,8$
Como se puede ver el mayor número de calificaciones cae dentro del rango que va desde 9.7 puntos hacia arriba hasta 7.8 puntos hacia abajo; puesto que la desviación estándar 0.94 representa el grado de dispersión que existe entre los valores de la variable con la media aritmética.

\section{Análisis estadístico del pre test aplicado al grupo de control}

Tabla 142. Distribución de frecuencias O1

\begin{tabular}{cccccc}
\hline$O_{1_{i}}$ & fi & hi & $O_{1_{i}} * f i$ & $O_{1_{i}}^{2}$ & $O_{1_{i}}^{2} * f i$ \\
\hline 3 & 5 & 0,17 & 15 & 9 & 45 \\
4 & 10 & 0,33 & 40 & 16 & 160 \\
5 & 10 & 0,33 & 50 & 25 & 250 \\
7 & 4 & 0,13 & 28 & 49 & 196 \\
8 & 1 & 0,03 & 8 & 64 & 64 \\
\hline TOTALES & 30 & 1,00 & 141 & 163 & 715 \\
\hline
\end{tabular}

Fuente: Secretaría del Centro de Idiomas ESPOCH

1. Cálculo de los estadígrafos

- Media Aritmética (Promedio $\mathrm{O}_{1}$ )

$\overline{\mathrm{O}_{1}}=\frac{\sum_{\mathrm{i}=1}^{\mathrm{n}} \mathrm{O}_{1_{\mathrm{i}}} * \mathrm{fi}}{\mathrm{n}}$

$\overline{\mathrm{O}_{1}}=\frac{141}{30}$

$\overline{\mathrm{O}_{1}}=4,7$

- Desviación Estándar

La desviación estándar nos permite conocer el grado de dispersión de los datos en relación a la media aritmética.

$\mathrm{S}_{\mathrm{O}_{1}}=\sqrt{\frac{\sum \mathrm{fi} * \mathrm{O}_{1_{\mathrm{i}}}^{2}-\frac{\left(\sum \mathrm{fi} * \mathrm{O}_{1_{\mathrm{i}}}\right)^{2}}{\mathrm{n}}}{\mathrm{n}-1}}$

$\mathrm{S}_{\mathrm{O}_{1}}=\sqrt{\frac{715-\frac{(141)^{2}}{30}}{30-1}}$

$\mathrm{S}_{\mathrm{O}_{1}}=\sqrt{1,80344}$

$\mathrm{S}_{\mathrm{O}_{1}}=1,3429$

2. Construcción de la Distribución de frecuencias $\mathrm{O}_{3}$ 
Tabla 15. Distribución de frecuencias de O3

\begin{tabular}{cccccc}
\hline$O_{3_{i}}$ & $\mathrm{fi}$ & $\mathrm{Hi}$ & $O_{3_{i}} * f i$ & $O_{3_{i}}^{2}$ & $O_{3_{i}}^{2} * f i$ \\
\hline 3 & 4 & 0,17 & 12 & 9 & 36 \\
4 & 10 & 0,43 & 40 & 16 & 160 \\
5 & 3 & 0,13 & 15 & 25 & 75 \\
6 & 2 & 0,09 & 12 & 36 & 72 \\
7 & 4 & 0,17 & 28 & 49 & 196 \\
\hline TOTALES & 23 & 1,00 & 107 & 135 & 539 \\
\hline
\end{tabular}

Fuente: Secretaría del Centro de Idiomas ESPOCH

3. Cálculo de los Estadígrafos

- $\quad$ Media Aritmética (Promedio $\mathrm{O}_{3}$ )

$\overline{\mathrm{O}_{3}}=4,65217$

$$
\begin{aligned}
& \overline{\mathrm{O}_{3}}=\frac{\sum_{\mathrm{i}=3}^{\mathrm{n}} \mathrm{O}_{\mathrm{i}_{3}}}{\mathrm{n}} \\
& \overline{\mathrm{O}_{3}}=\frac{107}{23}
\end{aligned}
$$

- Desviación Estándar de $\mathrm{O}_{3}$

$$
\begin{aligned}
\mathrm{S}_{\mathrm{O}_{3}} & =\sqrt{\frac{\sum \mathrm{fi} * \mathrm{O}_{3}^{2}-\frac{\left(\sum \mathrm{fi} * \mathrm{O}_{3}^{2}\right)^{2}}{\mathrm{n}}}{\mathrm{n}-1}} \\
\mathrm{~S}_{\mathrm{O}_{3}} & =\sqrt{\frac{539-\frac{107^{2}}{23}}{22}} \\
\mathrm{~S}_{\mathrm{O}_{3}} & =1,36876
\end{aligned}
$$

Del análisis del Pre-test a ambos grupos se obtiene la información que se presenta en la siguiente tabla.

Tabla 16. Resumen estadígrafos pre test

\begin{tabular}{lccc}
\hline & Pre Test & $O_{1}$ & $O_{3}$ \\
\hline Estadígrafo & & 4,7 & 4,65217 \\
Promedio & & 1,3429 & 1,3688 \\
Desviación Estándar & 30 & 23 \\
No. de Estudiantes & & 30 \\
\hline
\end{tabular}

Fuente: Secretaría del Centro de Idiomas ESPOCH

Para poder determinar si entre los dos grupos se pueden hallar diferencias significativas en el conocimiento de los temas pre vistos en los contenidos y conocimientos de los estudiantes previo al proceso experimental, se utilizó un estimador puntual éste permitió identificar un punto crítico para la diferencia de medias llamado cota de error.

Cálculo del estimador $\left(\overline{\mathrm{O}_{1}}-\overline{\mathrm{O}_{3}}\right)=0.04783$

La desviación estándar de medias es $\sigma\left(\overline{\mathrm{O}_{1}}-\overline{\mathrm{O}_{3}}\right)=\sqrt{\frac{\sigma_{\overline{\mathrm{O}_{1}}}^{2}}{\mathrm{n}_{\overline{\mathrm{O}_{1}}}}+\frac{\sigma_{\overline{\mathrm{O}_{3}}}^{2}}{\mathrm{n}_{\overline{\mathrm{O}_{3}}}}}$ 


$$
\begin{gathered}
\sigma\left(\overline{\mathrm{O}_{1}}-\overline{\mathrm{O}_{3}}\right)=\sqrt{\frac{1,3429^{2}}{30}+\frac{1,3688^{2}}{23}} \\
\sigma\left(\overline{\mathrm{O}_{1}}-\overline{\mathrm{O}_{3}}\right)=\sqrt{\frac{1,80}{30}+\frac{1,87}{23}} \\
\sigma\left(\overline{\mathrm{O}_{1}}-\overline{\mathrm{O}_{3}}\right)=0,38
\end{gathered}
$$

Siendo la cota de error $2\left(\sigma\left(\overline{\mathrm{O}_{2}}-\overline{\mathrm{O}_{4}}\right)\right)=0,75$

En esta cota se determina la decisión en que "si la diferencia de medias es mayor que la cota de error, entonces hay diferencia entre los promedios de las muestras; caso contrario se afirmaría que no hay diferencia significativa en el promedio de las muestras", como se puede observar la cota de error 0.753 es mayor a la diferencia de medias 0.38 entonces, se puede afirmar que los estudiantes no tienen diferencias significativas en los promedios obtenidos en el pre test, con lo que se inicia el experimento en igualdad de condiciones.

\section{Análisis estadístico del post test para la verificación de hipótesis}

Este análisis permite verificar las hipótesis planteadas en la investigación. Para este fin se desarrollaron cálculos estadísticos correspondientes a la prueba de hipótesis para muestras pequeñas. Los procedimientos seguidos son similares a los usados en el pre test, solo que en este caso aplicamos el contraste de hipótesis, así:

1. Distribución de frecuencias de $\mathrm{O}_{2}$

Tabla 17. Distribución de frecuencias de O2

\begin{tabular}{cccccc}
\hline$O_{2_{i}}$ & $\mathrm{fi}$ & $\mathrm{hi}$ & $O_{2_{i}} * f i$ & $O_{2_{i}}^{2}$ & $O_{2_{i}}^{2} * f i$ \\
\hline 4 & 6 & 0,20 & 24 & 16 & 96 \\
5 & 9 & 0,30 & 45 & 25 & 225 \\
6 & 9 & 0,30 & 54 & 36 & 324 \\
7 & 5 & 0,17 & 35 & 49 & 245 \\
8 & 1 & 0,03 & 8 & 64 & 64 \\
\hline TOTALES & 30 & 1,00 & 166 & 190 & 954 \\
\hline
\end{tabular}

Fuente: Secretaría del Centro de Idiomas ESPOCH

2. Cálculo de estadígrafos

- Media Aritmética (Promedio $\mathrm{O}_{2}$ )

$\overline{\mathrm{O}_{2}}=\frac{\sum_{\mathrm{i}=1}^{\mathrm{n}} \mathrm{O}_{1_{\mathrm{i}}} * \mathrm{fi}}{\mathrm{n}}$

$\overline{\mathrm{O}_{2}}=\frac{166}{30}$

$\overline{\mathrm{O}_{2}}=5,5333$ 
- Desviación Estándar

$$
\begin{aligned}
\mathrm{S}_{\mathrm{O}_{2}} & =\sqrt{\frac{\sum \mathrm{fi} * \mathrm{O}_{2_{\mathrm{i}}}^{2}-\frac{\left(\sum \mathrm{fi} * \mathrm{O}_{2_{\mathrm{i}}}\right)^{2}}{\mathrm{n}-1}}{\mathrm{~S}_{\mathrm{O}_{2}}}}=\sqrt{\frac{954-\frac{(166)^{2}}{30}}{30-1}} \\
\mathrm{~S}_{\mathrm{O}_{2}} & =1,1058
\end{aligned}
$$

- Varianza $\sigma_{\mathrm{O}_{2}}^{2}=1,22$

3. Distribución de frecuencias de $\mathrm{O}_{4}$

Tabla 183. Distribución de frecuencias de O4

\begin{tabular}{cccccc}
\hline$O_{4_{i}}$ & $\mathrm{fi}$ & $\mathrm{hi}$ & $O_{4_{i}} * f i$ & $O_{4}^{2}$ & $O_{4_{i}}^{2} * f i$ \\
\hline 6 & 1 & 0,04 & 6 & 36 & 36 \\
7 & 1 & 0,04 & 7 & 49 & 49 \\
8 & 5 & 0,22 & 40 & 64 & 320 \\
9 & 12 & 0,52 & 108 & 81 & 972 \\
10 & 4 & 0,17 & 40 & 100 & 400 \\
\hline TOTALES & 23 & 1,00 & 201 & 330 & 1777 \\
\hline
\end{tabular}

Fuente: Secretaría del Centro de Idiomas ESPOCH

4. Cálculo de los Estadígrafos

- Media Aritmética (Promedio $\mathrm{O}_{4}$ )

$\overline{\mathrm{O}_{4}}=\frac{\sum_{\mathrm{i}=1}^{\mathrm{n}} \mathrm{O}_{4 \mathrm{i}} * \mathrm{fi}}{\mathrm{n}}$

Tabla 4. Resumen estadígrafos post test

\begin{tabular}{cccccc}
\hline$O_{4_{i}}$ & $\mathrm{fi}$ & $\mathrm{hi}$ & $O_{4_{i}} * f i$ & $O_{4}^{2}$ & $O_{4_{i}}^{2} * f i$ \\
\hline 6 & 1 & 0,04 & 6 & 36 & 36 \\
7 & 1 & 0,04 & 7 & 49 & 49 \\
8 & 5 & 0,22 & 40 & 64 & 320 \\
9 & 12 & 0,52 & 108 & 81 & 972 \\
10 & 4 & 0,17 & 40 & 100 & 400 \\
\hline TOTALES & 23 & 1,00 & 201 & 330 & 1777 \\
\hline
\end{tabular}

Fuente: Secretaría del Centro de Idiomas ESPOCH

$$
\begin{aligned}
& \overline{\mathrm{O}_{4}}=\frac{201}{23} \\
& \overline{\mathrm{O}_{4}}=8,7391
\end{aligned}
$$


- Desviación Estándar de $\mathrm{O}_{4}$

$$
\begin{aligned}
& \mathrm{S}_{\mathrm{O}_{4}}=\sqrt{\frac{\sum \mathrm{fi} * \mathrm{O}_{4_{\mathrm{i}}}^{2}-\frac{\left(\sum \mathrm{fi} * \mathrm{O}_{4_{\mathrm{i}}}\right)^{2}}{\mathrm{n}-1}}{\mathrm{n}}} \\
& \mathrm{S}_{\mathrm{O}_{4}}=\sqrt{\frac{1777-\frac{(201)^{2}}{23}}{23-1}} \\
& \mathrm{~S}_{\mathrm{O}_{4}}=0,9637 \\
& \text { - Varianza } \\
& \sigma_{\overline{\mathrm{O}_{4}}}^{2}=0,93
\end{aligned}
$$

Del análisis del post test a los grupos de control y experimental se obtiene la información que se detalla en la siguiente tabla.

La desviación estándar de la diferencia de medias es $\left(\overline{\mathrm{O}_{2}}-\overline{\mathrm{O}_{4}}\right)=3,2$

Siendo la cota de error $2\left(\sigma\left(\overline{\mathrm{O}_{2}}-\overline{\mathrm{O}_{4}}\right)\right)=0,569$

En esta cota se determina la decisión en que "si la diferencia de medias es mayor que la cota de error, entonces hay diferencia entre los promedios de las muestras, caso contrario se afirmaría que no hay diferencia significativa en los promedios de las muestras" como se puede observar la cota de error 0.569 es menor a la diferencia de medias 3.2, se puede afirmar que los estudiantes tienen diferencias significativas en los promedios del post test.

La validación se la realizó con el paralelo "G" del Segundo Nivel del Centro de Idiomas de la Escuela Superior Politécnica de Chimborazo. Como instrumento para este evento se utilizó Alfa de Cronbach que es un coeficiente que sirve para medir la fiabilidad de una escala de medida.

$$
\begin{array}{cc} 
& \alpha=\frac{\mathrm{k}}{\mathrm{k}-1}\left[1-\frac{\sum \mathrm{S}_{\mathrm{i}}^{2}}{\mathrm{~S}_{\mathrm{T}}^{2}}\right] \\
\text { K } & 60 \\
\text { SUM.VAR } & 9.6172414 \\
\text { V.T. } & 73.389655 \\
\text { SECCIÓN 1 } & 1.0169492 \\
\text { SECCIÓN 2 } & 0.8689564 \\
\text { ALFA DE CRONBACH } & 0.8836845
\end{array}
$$


El nivel de confianza obtenido 0.88368454 aproximado a una cifra decimal es 0.9 y como éste está por encima de 0.7 que es punto de referencia el instrumento de evaluación es una herramienta que ha sido aceptada por tener un nivel de confianza aceptado.

\section{Conclusiones}

- La estrategia Chunking Flashcards puede ser enseñada en cualquier nivel de estudio. Esta estrategia permite descomponer una palabra en otras derivadas facilitando la reproducción, el almacenamiento en la memoria y la evocación de vocabulario en situaciones de aprendizaje.

- El nivel de competencia léxica está determinado por el rango de las calificaciones obtenidas en el Post-test que elevó la medida de 5,5 a 8,7, porque los Chunking Flashcards como ayuda visual favorecen en los estudiantes siendo su aprendizaje más significativo, permitiéndoles la fijación permanente en la memoria y reduciendo el tiempo de respuesta sobre lo aprendido, aspecto reflejado en las respuestas al cuestionario aplicado en el Post-test.

- El nivel de competencia está determinado por la diferencia de medias de las calificaciones entre el Grupo de Control (Gc) y la media de las calificaciones del Grupo Experimental $(\mathrm{Ge})$. Al existir diferencias significativas entre los datos obtenidos de la enseñanza tradicional y la enseñanza utilizando la estrategia Chunking Flashcards se puede concluir que el uso de los Chunking Flashcards incide favorablemente en la adquisición de la competencia léxica con los estudiantes del Segundo Nivel de Idiomas de la Escuela Superior Politécnica de Chimborazo.

- El Chunking reduce el tiempo dedicado al aprendizaje y amplia el vocabulario como parte de la competencia léxica, debido a que la estrategia Chunking Flashcards ayudó a organizar los elementos en grupos significativos, en donde en lugar de percibir letras aisladas, percibieron y recordaron su agrupación significativa ayudada a través de las Flashcards

- Por último, se concluye que en el desarrollo de la intervención durante el proceso de experimentación con la aplicación de la estrategia Chunking Flashcards como una nueva tendencia en la enseñanza del idioma ingles se evidenció un alto nivel de motivación y mayor participación interactiva docente Chunking estudiante, que a más de incrementar su competencia lexical mejoro el interés por aprender el idioma inglés.

\section{Referencias}

Alba, Q. V. (2011). La Competencia Lexica: Una propuesta de actividades sobre los campos lèxicos para clases de ELE. Revista de Didactica Española como lengua extranjera. Universidad de Pablo de Olavide.

AllenNewell. (1990). Chunking in Soar. Machine learning, 11/46.

Amaguaña, K. (2012). Competencia Comunicativas. Esmeraldas.

Ansoft, H. 1. (11 de 03 de 1976). Gestiopolis.com. Obtenido de https://www.gestiopolis.com/un-concepto-de-estrategia/ 
Apostilla. (2014). "Concepto definiciones. Educacion P -definista.

Baugh, A. C And Cable, T. (2002). A History of the English Language. Recuperado el 23 de 02 de 2019, de Facltity .mu.edu.sa: http://faculty.mu.edu.sa

Bernal, B. J. (2010). Metodo y Metodogia en la Investigacion Cientifica. Guayaquil: Estilo de vida. Guayaquil: Estilo de vida.

Bogaards, P. (1996). Lexicon and grammar in second language learning" en P. Jordens y J. Calleman (eds.) Investigating Second Language Adquisition, . Berlin: Gruyter pp. 357-379.

Chadwick.

(1996).

Blogspot.com.

Obtenido

de http://psicojeffestrategiascognitivas.blogspot.com/

Chomsky, N. (1987). Estructuras Sintácticas. Editorial Siglo XXI.

Cicarelli, M. C. (10 de 12 de 2018). Estrategias-Cognitivas. Obtenido de EstrategiasCognitivas: https://www.psicopedagogia.com/

Colmenarez, J. (29 de 04 de 2015). Ingles corocito:. Obtenido de inglescorocito.blogspot.com: http://inglescorocito.blogspot.com

Crystal, D. (1997). English as a global language. : . Cambridge: University Press.

Dansereau. (1986). Que son las estrategias de aprendizaje. Cientifica Universsitaria. 315.

Ellis. (1996). Prospective memory. New York and London: Psychology Press.

Fernández, O., Martínez-Conde, M. \& Melipillán, R. (2009). Estrategias de aprendizaje y Autoestima. Su relación con la permanencia y deserción Universitaria (Vol. Vol.1.). Revista de Estudios Pedagógicos.

García, M. H. (2006). Estudio de las colocaiones léxicas y su enseñanza en español como lengua extranjera. . Málaga: Coleccion Monográs.

Hernández, S. R. (2003). Metodología de la Investigación. . México: Mc Graw Hill.

Importancia.org. (15 de 11 de 2015). Obtenido de https://www.importancia.org/ingles.php

Izquierdo, G. M. (2004). La seleccion del lexico en la enseñanza del español como lengua extranjera. Valencia,: http://www.tdx.cesca.es/TDX-0425105-135323. Recuperado el 23 de 02 de 2019, de http://www.tdx.cesca.es

Kahneman, O., Cabestrero Raúl. (2013). Fundamentos Psicológicos de la Actividad Cardiovascular y oculomotora. Madrid: Editorial digital.

Lightbown, Patsy M.; Nina Spada. . (1993). How languages are learned. Oxford: University Press.

Maley, A. (2004: 7). Los procesos sensoriales de la experiencia y el vocabulario". Resource Books for Teachers. Oxford., 7.

Marco Comùn Europeo de Referencia. (2002). Para lenguas, parendizaje, enseñanza, evaluaciòn. 108.

Mathias, P. (2001). The First Industrial Nation: The Economic History of Britain 17001914.Londres: Routledge. Londres: Routledge.

Mercedes. (2010). Competencia Lexica". Espanol en America.

Miller, G. A. (1990). The pleace of lenguage in a scientific psychology. Massachuseth: Psychological Science.

Ministerio de Educación, C. y. (2002). Marco común europeo de referencia para las lenguas: aprendizaje, enseñanza evaluación. Madrid: Anaya. 
Morante Vallejo, R. (2005). El desarrollo del conocimiento léxico en segundas lenguas,. Madrid: Arco/libros.

Muñoz, C. (2002). Aprender idiomas. Barcelona: Paidós.

Newell., A. (1990). Chunking in Soar. Machine learning.

Núñez, .. M. (2010). Competencia Lexica, Competencia Comunicativa. Departamento de Didáctica de la Lengua y la Literatura Universidad de Granada, 91:97.

Ochaita, E. (1988). Aspectos Cognitivos del Desarrollo Psicológico de los Ciegos (II). En E. O. otros. Madrid: Gràficas Juma.

Peralta, W. (2016). Estrategias de Ensenanza aprendizaje del Idioma como lengua extranjera. Revista vinculando.

Pérez Basanta, C. (1999). La enseñanza del vocabulario desde una perspectiva lingüística y pedagógica”. En S. Salaberri (ed.). Lingüística Aplicada a las Lenguas Extranjeras (pp. 262-307). Almería: Universidad de Almería.

Piaget, J. (1931). El lenguaje y el Pensamiento en el Niño. Barcelona: Editorial Paidos Iberica.

Rodriguez, M., García, F., \& García, A. (10 de 2017). Pretest y Postest. Salamanca: GRIAL. Obtenido de http://sociologianecesaria.blogspot.com

Skinner, B. (1957). Conducta Verbal. Acton. Massachusetts: Copley Publishing Group.

Velasquez, M. (09 de 07 de 2013). Goconqr.com. Obtenido de https://www.goconqr.com

Welers, R. (1986). Investitgación de Mercados. México: Ed. Prentice Hall 96-137.

Wright, S. (2004). Language policy and language planning: From nationalism to globalization. Basingstoke: Palgrave.

Zabert, A. (2010). PIIS Escuela Leloir. Obtenido de Estrategias de Aprendizaje.

Zimmerman, C. (1997). Historical trends in second language vocabulary instruction" en J, Coady y T. N. Huckin (eds.) Second Language Vocabulary Acquisition: A Rationale for Pedagogy. Cambridge: : CUP (pp. 5-19). 


\section{PARA CITAR EL ARTÍCULO INDEXADO.}

Herrera Andrade, Z., Barragán Murillo, R. de los Ángeles, Colcha Guashpa, E., \& Salazar Calderón, E. (2019). Competencia léxica en inglés mediante el uso de la Estrategia Chunking $\begin{array}{llll}\text { Flashcards. } & \text { Ciencia } & \text { Digital, } & \text { 3(3.2), }\end{array}$ https://doi.org/10.33262/cienciadigital.v3i3.2.715

\section{Liencia}

El artículo que se publica es de exclusiva responsabilidad de los autores y no necesariamente reflejan el pensamiento de la Revista Ciencia Digital.

El artículo queda en propiedad de la revista y, por tanto, su publicación parcial y/o total en otro medio tiene que ser autorizado por el director de la Revista Ciencia Digital.
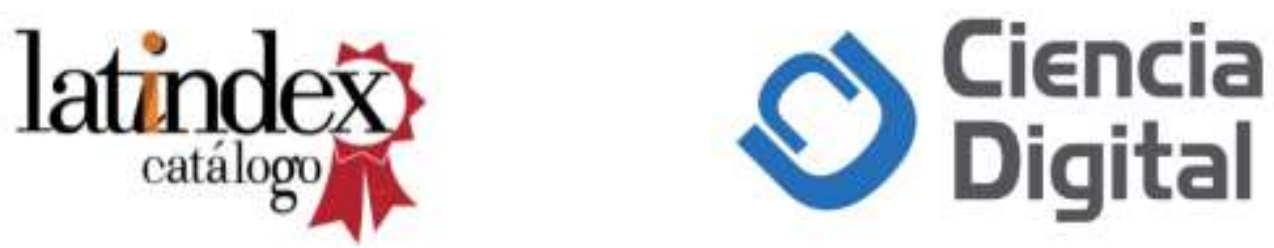